\title{
Erfolgsmotor Value Selling
}

Value Selling wird in vielen Unternehmen und Vertriebspublikationen gefordert. Alle reden davon, doch jeder versteht darunter etwas anderes und nur selten wird es konsequent umgesetzt. Um Value Selling im Bereich Business-to-Business zu schärfen und zu verwirklichen, ist ein systematischer Ansatz notwendig.

Christian Belz, Holger Dannenberg 
Unsere zentrale These lautet: 80 Prozent der Unternehmen verkaufen ihre Leistungen unter ihrem Wert. Das muss mit Value Selling verbessert werden.

Wie wichtig ist Value Selling für Unternehmen? 87 Prozent der Unternehmen bezeichnen laut einer Umfrage, die wir 2015 unter 278 Praktikern durchgeführt haben, Value Selling für ihr Unternehmen als zentrales Thema. Offensichtlich geht es nicht nur darum, den Ansatz zu bezeichnen, sondern ihn stetig besser als Wettbewerber für Kunden umzusetzen. Mit professionellem Value Selling können Unternehmen ihre Verkaufspreise im Durchschnitt um 14 Prozent steigern. Ihr Potenzial ist für Value Selling aber erst zu 31 Prozent ausgeschöpft. Auch geht nahezu die Hälfte der Initiativen für eine umfassendere Zusammenarbeit von den Kunden aus, sie sitzen also auf dem „Fahrersessel“. In 34 Prozent der Fälle stoßen die Anbieter immer wieder auf die gleichen Wettbewerber. Deshalb ist es nötig, das eigene Vorgehen im Verkauf grundsätzlich zu klären.

\section{„Mit professionellem Value Selling können Unternehmen ihre Verkaufspreise im Durchschnitt um 14 Prozent steigern."}

Wir definieren Value Selling als eine Verbesserung der Verkaufsprozesse und der Interaktion mit Kunden. Es verbindet das eigene Angebot konsequent mit der Sichtweise und dem Nutzen des Kunden.

Vielfältige Begriffe wie Nutzenverkauf, Value Proposition, Lösungsverkauf, strategischer Verkauf, Mastering the Complex Sale, Spin Selling, Professional Sales, Sales Excellence, Challenger Sale, Experience Selling, Adaptive Selling, Consultative Selling, Performance Selling oder Enterprise Selling weisen in ähnlicher Richtung oder betonen einzelne Aspekte.

\section{Roadmap zu Value Selling}

Wie ist ein Projekt im Unternehmen für Value Selling anzugehen? Wir unterscheiden in Abbildung 1 vier Schritte, die sich allerdings auch gegenseitig bedingen. Die konkrete Roadmap oder auch Projektpläne gilt es, auf das eigene Unternehmen anzupassen.

Die einzelnen Schritte beschreiben wir kurz in der Folge.

\section{Schritt 1: Voraussetzungen der Leistung schaffen und Ziele für Value} Selling vorgeben

Der Wert für Kunden beruht auf der Leistungsfähigkeit des Unternehmens. Typische Fragen sind beispielsweise: Wie gelingt es, unterschiedliche Produkte und Module für Kunden zu kombinieren? Wie effizient arbeitet das Unternehmen mit Kunden zusammen? Welchen Kundenbedürfnissen wird mit herausragenden Services begegnet? Wie wirtschaftlich ist

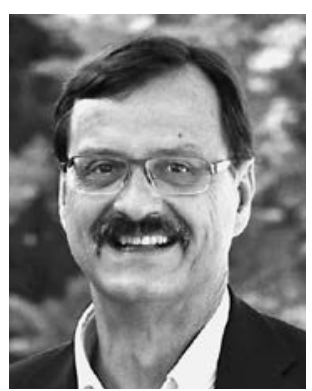

Prof. Dr. Christian Belz

ist Ordinarius für Marketing an der Universität St. Gallen und Direktor am Institut für Marketing.

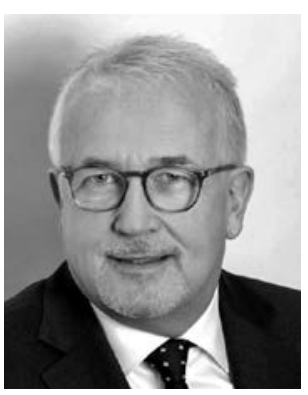

\section{Holger Dannenberg} ist Geschäftsführer Deutschland von Mercuri International und als Global Partner im internationalen Geschäft von Mercuri International eingebunden. 


\section{Zusammenfassung}

- Was heute im Value Selling einzigartig ist, wird morgen bereits selbstverständlich sein. Deshalb ist Value Selling ein permanentes „Kampfthema“.

- Die Schritte für erfolgreiches Value Selling sind: 1. Leistungsvoraussetzungen des Unternehmens schaffen und operationale Ziele des Value Selling vorgeben, 2. Value Selling fokussieren, 3. Value Selling in Verkaufsgespräche umsetzen und 4. Value Selling als Change Prozess führen.

das Angebot? Der Verkauf kann keine Werte verkaufen, die der Anbieter nicht leistet. Damit verlängert Value Selling erst die Strategie des Unternehmens für Leistungen und Kunden, setzt sie um.

Ziel des Value Selling ist, die eigene Leistung nach ihrem Wert für Kunden zu verkaufen. Dazu gilt es, die Mehrwerte für
Kunden sichtbar und wichtig zu machen. Es geht darum, das Werterlebnis des Kunden zu steigern. Abbildung 2 zeigt, wie Unternehmen ihre Ziele mit Value Selling gewichten.

Die Ergebnisse zeigen die Zielbereiche für Value Selling. Sie werden im Unternehmen in operationale Vorgaben umgesetzt. So spielt es beispielsweise eine Rolle, ob ein Anbieter mit Cross Selling zehn oder 30 Prozent wachsen will oder der Anteil von neuen Kunden für das nächste Jahr von zehn auf 15 oder 30 Prozent gesteigert werden soll.

Ziele lassen sich auch „bottom up" aus der erfolgreichen $\mathrm{Zu}$ sammenarbeit mit einzelnen Kunden ableiten. Damit ist Ausgangspunkt, was bereits funktioniert.

\section{Schritt 2: Value Selling fokussieren:}

\section{Delta des Verkaufsapproaches und Kundenvorteile}

Value Selling kann sich auf verschiedene Veränderungen und Initiativen richten, wie sie Abbildung 3 zeigt. Die Signete bezeichnen Unternehmen, die beim einzelnen Thema besonders ansetzten. Die verschiedenen Ausrichtungen wurden in unserer Befragung gewichtet.

\section{Abb. 1 Vier Schritte für Value Selling}

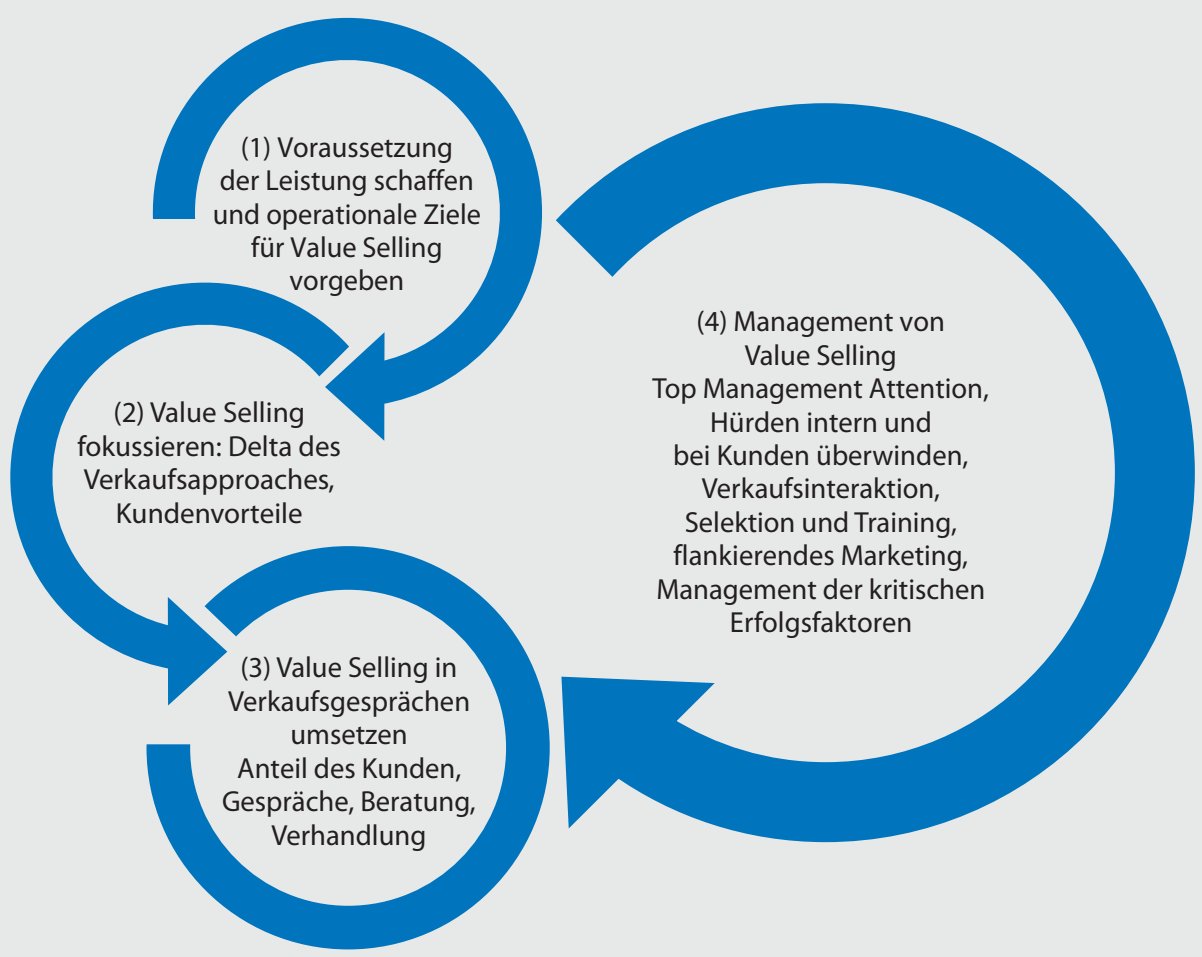


Es kann nicht Ziel sein, alle Ansätze zu verfolgen. Im Gegenteil: Value Selling wird in Unternehmen zu breit angesetzt und verwässert.

\section{Entscheider ausgewogen berücksichtigen}

Bereits eine angestrebte Verbesserung fordert die Unternehmen heraus. Nehmen wir beispielsweise „vom Einkäufer zum Buying Center“. Oft verschieben sich die wichtigen Entscheider im Unternehmen. Beispielsweise sind Top-Management und Bereichsleiter stärker für Beschaffungen der Informatik zuständig oder im Spital verlagert sich die Einkaufsmacht vom medizinischen Bereich zu zentralen Einkäufern. Schließlich wollen Anbieter oft einen Zugang zum Top-Management gewinnen, um ihre umfassenden Lösungen wirksam einzubringen. Solche neuen Bezugspersonen erfolgreich anzusprechen, braucht neue Lösungen, Prozesse, Argumente und Anlässe. Begleitet werden diese Anstrengungen dadurch, dass die wichtigen Entscheider und Beeinflusser in dynamischen $\mathrm{Bu}-$ ying Centers ausgewogen berücksichtigt werden. So kann es auch ergiebig sein, die zahlreichen Nutzer von Materialien und Anlagen in Marketing und Vertrieb zu gewinnen. Die Anforderungen an den Verkauf, aber auch weiterer Personen im Unternehmen, werden damit maßgeblich erweitert.

Strategisch setzt das Unternehmen die Schwerpunkte bei angestrebten Vorteilen für Segmente oder Kundengruppen.
Für jeden spezifischen Austausch mit einem Kunden prüft zudem der Verkäufer, welche Vorteile er gezielt einbringen soll. Sind es Vorteile im Image des Anbieters, in der persönlichen Beziehung, in der herausragenden Erklärung der Leistung, in der Individualisierung der Leistung, in der Entlastung und Sicherheit des Kunden; im Kundenbeitrag des Anbieters zu sei-

\section{„Wird Value Selling als umfassende Initiative verstanden und über mehrere Jahre verfolgt, so wirkt es stark und nachhaltig."}

ner Qualität, Innovation, Wirtschaftlichkeit, Geschwindigkeit und Flexibilität oder liegt der besondere Vorteil in der effizienten Koordination im eigenen Unternehmen und beim Kunden? Selten ist es dabei wirksam, zu viele Argumente oder Vorteile einzubringen.

\section{Schritt 3: Value Selling in Verkaufsgesprächen umsetzen} Die Werte des Anbieters für Kunden gilt es gekonnt in Gesprächen, Offerten und Verhandlungen einzubringen. Was

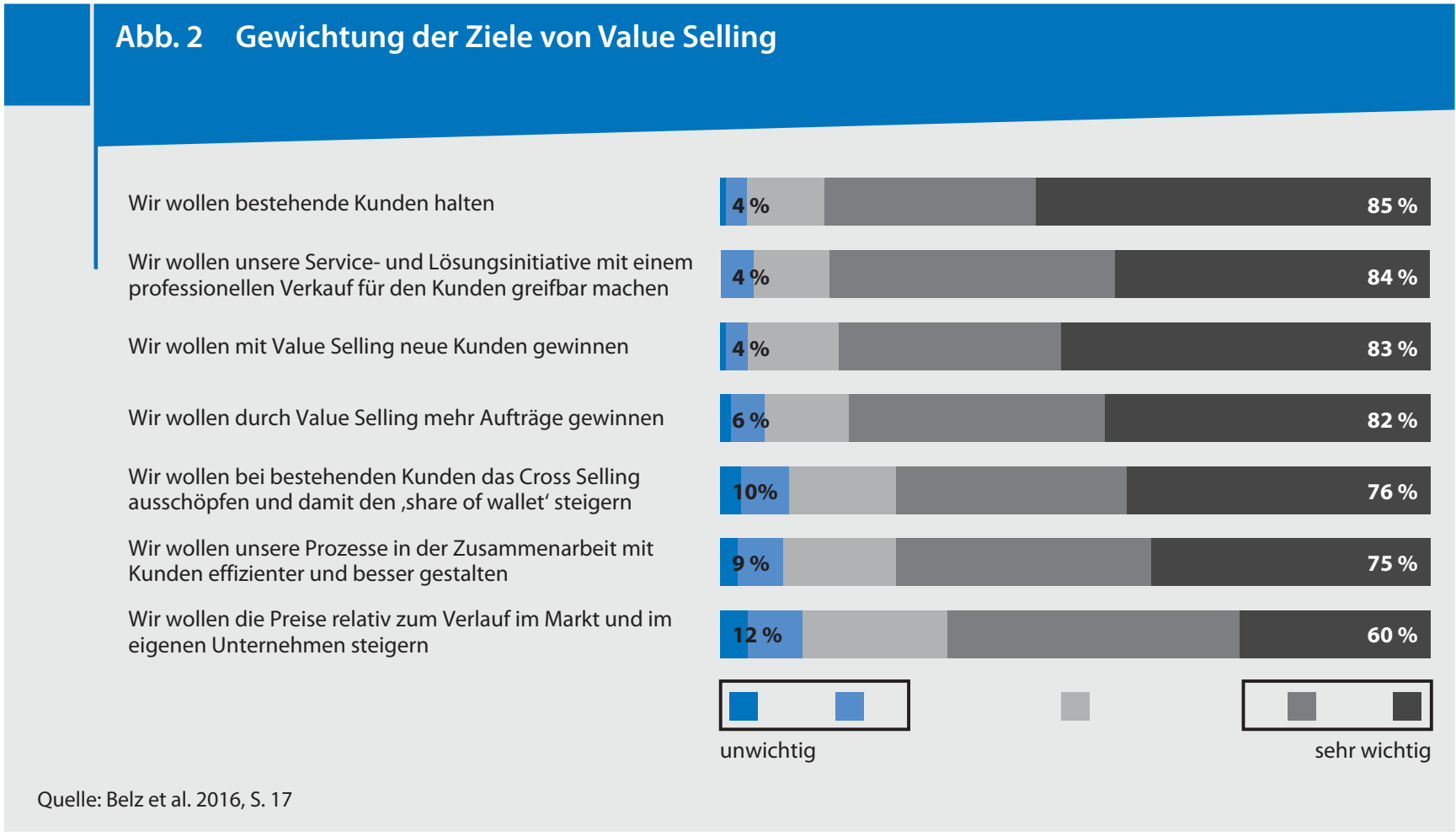


nicht in die persönliche und unpersönliche Interaktion mit Kunden fließt, findet nicht statt.

Wichtig ist es vorerst, die groben Fehler im Verkauf und Service zu vermeiden, die den Kunden zum Abbruch der Geschäftsbeziehungen führen. Dann bewegen sich manche Unternehmen in einer Verkaufsillusion. Sie delegieren dem Verkauf weit mehr Aufgaben, als dieser erfüllen kann. Bezug bleibt auch immer die Gesprächszeit mit Kunden, die tendenziell laufend verkürzt wird. Es ist nicht möglich, zehn Themen in 60 Minuten vernünftig aufzugreifen. Value Selling verändert die Interaktion mit Kunden. Spitzenreiter aus unserer Befragung waren dabei 360-Grad-Information zum Kunden, geschickte Preisgespräche und Value Pricing (im Vergleich zum Wettbewerb), proaktives Zuhören und Fragen sowie qualifizierte Beratung. Ergebnis kann dabei sein: Der Verkäufer wird zum „Trusted Adviser“ (Maister).

Jeff Thull bemerkt in seinem Buch „Mastering the Complex Sale", dass im Bereich Business-to-Business weit mehr als 35 Prozent der wahrgenommenen „Sales Opportunities“ im Sande bei

\section{Kerngedanken}

- Value Selling ist ein Erfolgsmotor. Der Ansatz bringt anspruchsvolle Leistungs-/Markt-Strategien erst auf den Boden.

- Value Selling muss als Ansatz für das eigene Unternehmen fokussiert werden. Es gilt konkret zu bestimmen, was sich mit Value Selling bei Kunden und im eigenen Unternehmen verändern soll.

- Im Unternehmen und beim Kunden gibt es Widerstände, die es zu überwinden gilt.

Kunden verlaufen. Auch seien die Unsicherheiten bei Kunden unterschätzt. Sein Vorschlag: "Business-to-Business companies need a smarter way to bring their value to market and transform it into profitable growth. They need a platform that is specifically designed for the complex sales arena. ... Diagnostic Business De-

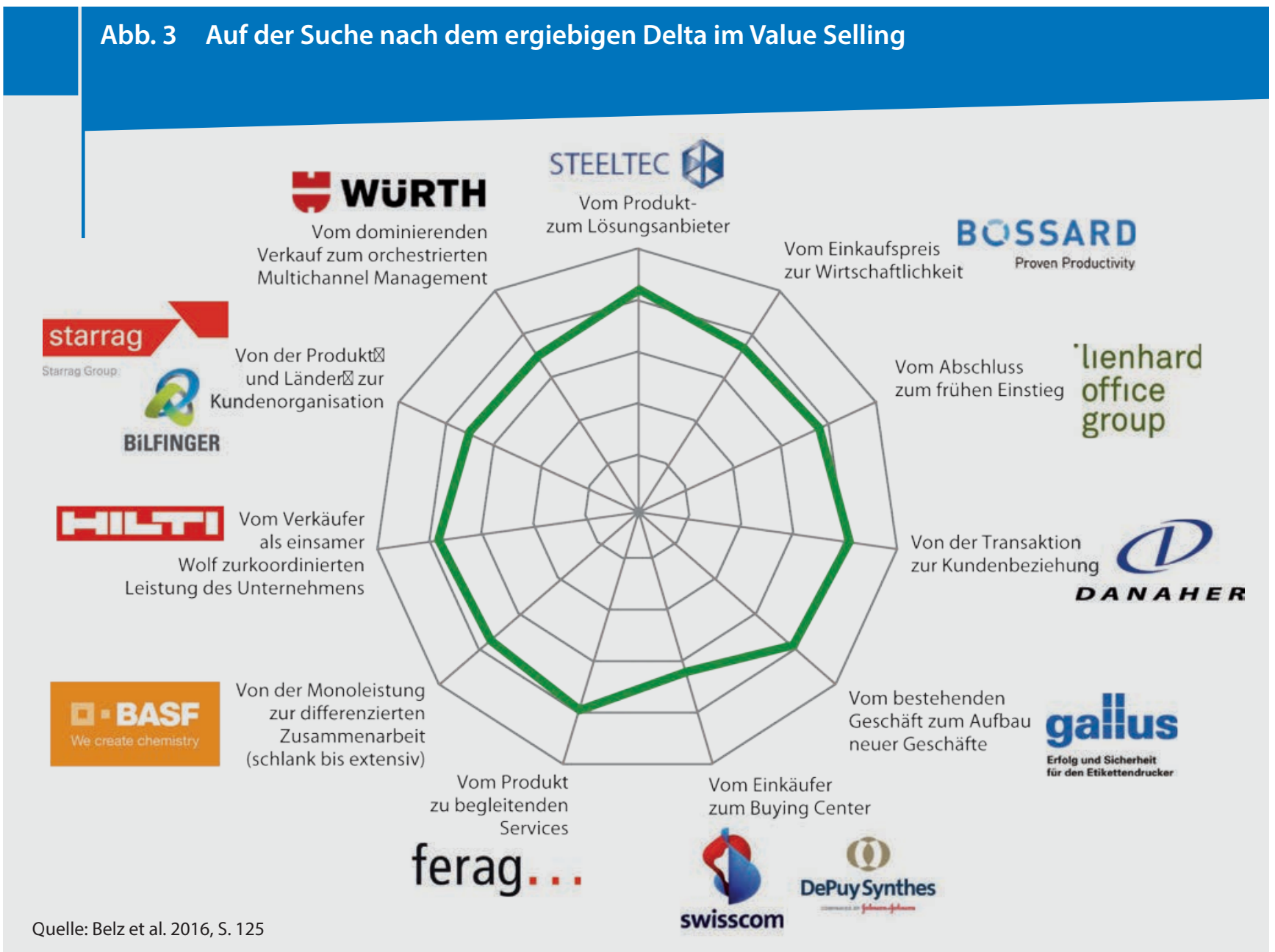


velopment is this smarter way to sell because it converts the conventional solution-based, seller-first approach into a diagnostic, customer first approach. It eliminates obsolete sales processes driven by premature presentations, debate, and confrontation, and replaces them with a step-by-step process of mutual confirmation between the sales team and the customer."

Interessant ist auch der Challenger-Sale-Ansatz von Brent und Dixon. Ihre Untersuchungen zeigen, dass besonders erfolgreiche Verkäufer ihre Kunden herausfordern, ihre Lösungen und das Vorgehen in Frage stellen oder völlig neue Wege vorschlagen. Dieser Mehrwert entwickelt eine gute und wichtige Beziehung zum Kunden. Konzerne wie SAP verfolgen den Ansatz mit eindrücklichen Ergebnissen bei den geschulten Verkäufern im Vergleich zum Rest: Sie gewannen mit der Initiative 26 Prozent mehr Verträge, steigerten den Umsatz um 27 Prozent, generierten 26 Prozent mehr Verkaufschancen, steigerten die Vertragssumme um den Faktor 6 und senkte die Zeit mit zu den Vertragsabschlüssen um 25 Prozent.

\section{Schritt 4: Management von Value Selling}

Das Management des Value Selling betrifft auch die Schritte 1 bis 3. Offensichtlich sind die Ansätze im Management vielfältig, wie unsere Untersuchungsergebnisse in Abbildung 4 verdeutlichen.

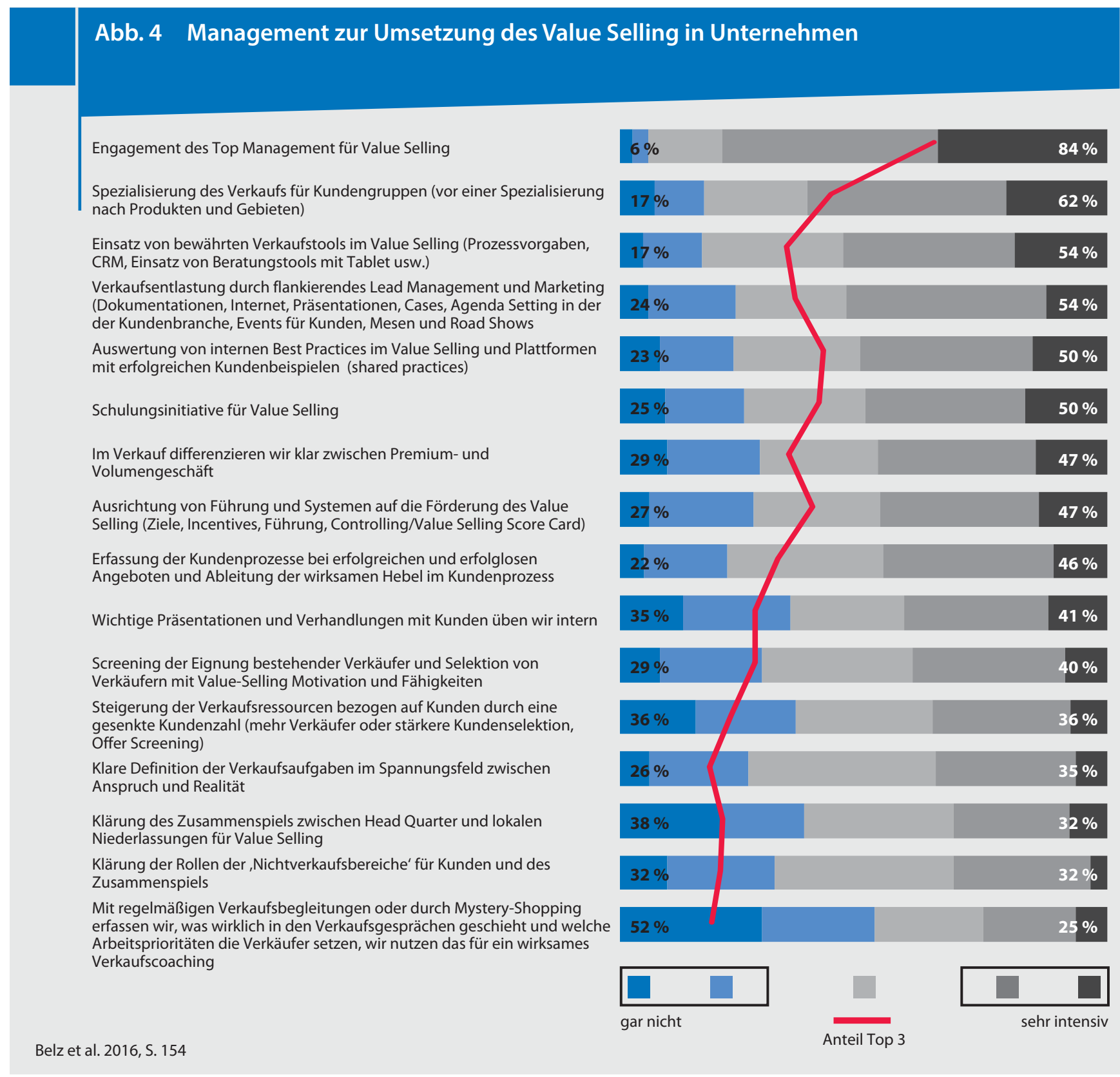




\section{Handlungsempfehlungen}

- Setzen Sie operationale Ziele für Value Selling und verfolgen Sie, wie weit Sie diese Ziele erreichen.

- Bestimmen Sie das Delta zwischen Ist und Soll im Value Selling.

- Übersetzen Sie Ihr Angebot in Kundenvorteile.

- Entwickeln Sie die Verkaufsgespräche mit Kunden.

- Führen Sie den Change Prozess im Unternehmen zu Value Selling.

Das Commitment des Top Management ist zentral. Auch scheint die Organisation des Verkaufs nach Kunden besonders ergiebig. Verkaufstools reichen von CRM, definierten Verkaufs-Kunden-Prozessen (z.B. Sales Funnel) bis zu (elektronischen) Anleitungen für strukturierte und kundenbezogene Gespräche. Das Controlling setzt an den formulierten Zielen (Schritt 1) an.

Im Change Management zum Value Selling gilt es interne Hürden (z.B. viel Kraft um interne Kräfte für Kunden zu mobilisieren oder falsche Lohnsysteme) und auch Hürden bei den Kunden (z.B. taktische Konzentration des Kunden auf Einkaufspreise bei gleichzeitig wachsenden Anforderungen oder falsche Zielvorgaben des Einkaufs) zu überwinden.

\section{Fazit}

Value Selling scheint in der Praxis und auch teilweise in der Forschung alles und nichts zu sein. Entsprechend wichtig ist es, den Ansatz für Unternehmen zu spezifizieren. Bereits zu Worthülsen in der Strategie wird applaudiert. Für konkrete Verbesserungen braucht es aber weit mehr.

Um Value Selling zu spezifizieren, dienen die festgestellten Deltas für mehr Verkaufsprofessionalität und auch die Kundenvorteile. Schließlich muss es gelingen, die angestrebten Veränderungen in die tausenden Verkaufsgespräche eines Unternehmens zu überführen und laufend zu entwickeln und den Change zum Value Selling zu managen. Wird Value Selling als umfassende Initiative verstanden und über mehrere Jahre verfolgt, so wirkt es stark und nachhaltig.

Treiber des Themas Value Selling sind Preisdruck (in der Schweiz durch die Wechselkursverschiebung Anfang 2015 akzentuiert), Globalisierung und neue Wettbewerber. Es gibt zudem in den meisten Branchen Vorbilder. Auch wollen Unternehmen ihre herausragenden Leistungen nicht unter ihrem
Wert verkaufen. Value Selling wird zu einem Erfolgs- bis Überlebensthema für Anbieter.

Jedes Unternehmen nutzt Value Selling. Gibt es eine Skala von 1 mit Anfängern bis zu 10 mit Benchmarks für die Branche, so gilt es, den eigenen Standort nach vorne zu treiben, also beispielsweise von $5 \mathrm{zu} 7$.

Kritische Themen aus der Befragung sind besonders: Wie gehen Anbieter mit dem Thema Wirtschaftlichkeit bei Kunden vor? Wie werden Buying Centers als Basis für Kundenlösungen genutzt? Wie fördern Unternehmen ihre Kundenorientierung im Verkauf? Und generell: Wie lässt sich Value Selling besser fokussieren? Nicht die Absichten und Ziele sind herausfordernd, sondern Weg und Geschwindigkeit.

Die Ansprüche an den persönlichen Kauf sind sehr hoch. Es entfacht sich deshalb ein wahrer, Krieg um die besten Verkaufstalente. Es gilt, fähige Verkäufer auf allen Stufen zu fördern. Value Selling muss bei den Verkäufern reifen, wer nur zwei Jahre auf dem Job bleibt hat keine Chance. Gleichzeitig müssen sie die hohe Wertschätzung für ihre Arbeit im Unternehmen spüren.

\section{Literatur}

Belz, Ch., Dannenberg, H., Redemann, M., Weibel, M. (2016): Value Selling, Stuttgart

Brent, A., Dixon, M. (2016): The Challenger Sale: Kunden herausfordern und erfolgreich überzeugen, 2. aktualisierte Auflage, Wien/ Frankfurt

Maister, D. et al. (2002): The Trusted Adviser, New York

Thull, J. (2010): Mastering the Complex Sale, Second Edition, Hoboken N.J.

Stern, R. (2016): Die Erneuerung des Vertriebs, in: Harvard Business Manager, Februar 2016, S. 63-69

Weitere Empfehlungen der Verlagsredaktion aus www.springerprofessional.de zu:

\section{Value Selling}

Artmann, Ch.: Erfolgsfaktoren für den ínternationalen Roll-out von Value Selling, in: Marketing Review St. Gallen, Nr. 6/2014, S. 67-77, Wiesbaden 2014, www.springerprofessional.de/link/6402386

Kaschek, B.: True Value Selling: Umfeld und erste Ansätze, in: Kaschek, B.: True Value Selling, Wiesbaden 2014, www.springerprofessional.de/link/4286460 
„Das persönliche Verkaufen

hat sich nicht geändert"

\author{
Martin Schäfer, Geschäftsführer Vertrieb der Adolf Würth GmbH \& Co. KG, \\ sprach mit Christian Peters, Head of Market Development bei Mercuri International, \\ über die Umsetzung von Value Selling bei Würth.
}

\title{
Christian Peters
}

\section{Was heißt Value Selling für Würth?}

Wissen Sie, die Firma Würth hat sich besonders auf den Handel von C-Teilen spezialisiert. Die Artikel, die ein Kunde bei uns bezieht, will und kann er nicht den ganzen Tag im Kopf haben und sich zu viele Gedanken darüber machen. Und genau hier setzt unsere Strategie an. Wir versuchen nicht nur einem Kunden beispielsweise ein paar Schrauben zu verkaufen. Unser Konzept geht dahin, ihm die Sorge um den Nachschub und über ein leeres Lager zu nehmen. Unsere Produkte werden erst für den Kunden wichtig, wenn sie mal fehlen. Hier bieten wir ein breites Angebot an Systemen und automatisierten Services.

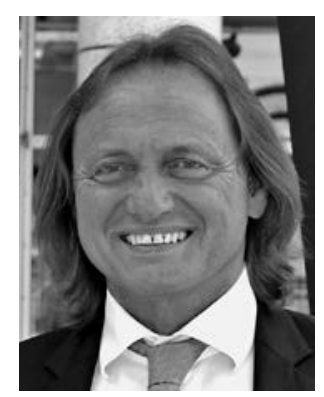

\section{Martin Schäfer}

ist Geschäftsführer Vertrieb der Adolf Würth $\mathrm{GmbH}$ \& Co. KG. Seine Karriere begann er als Außendienstmitarbeiter, durchschritt eine klassische Außendienstlaufbahn und stieg nach mehreren Stationen im Verkauf im Jahre 1993 zum Verkaufsleiter auf. Im Jahr 2000 wurde er als Geschäftsführer für den Vertrieb Deutschland ernannt.
Was sind für Sie die wesentlichen, inhaltlichen Bausteine für Value Selling?

Der Kunde steht ja besonders bei unseren kleinen Befestigungsprodukten oftmals vor der Frage, warum soll ich das bei Würth kaufen und nicht woanders. Hier versuchen wir dem Kunden ein „Gesicht“ zu Würth zu geben. Das ist dann eben der Verkäufer, der die Produkte, aber speziell auch die Marke Würth repräsentiert. So hat am Ende der Kunde, auch wenn er etwas über unseren E-Shop oder einen Abholshop bezieht, trotzdem das Gefühl von Sicherheit, da er weiß, wer „sein“ Würth ist.

Wie gestalten Sie eine wirksame Schulung in Value Selling? Wie unterstützen Sie den Transfer in die Praxis der Verkäufer? Wir haben schon vor vielen Jahren angefangen, unsere Verkäufer in der hauseigenen Akademie auszubilden. Hier wird natürlich besonders der Fokus auf den Produktnutzen und den Mehrwert für den Kunden unserer Services geschult, damit diese von Anfang an in den Vordergrund gestellt werden. Aber der Kundenbesuch ist natürlich der meisttrainierte Inhalt. Eine Beziehung zum Kunden auf- und ausbauen ist der Grundstein für Vertrauen und eine erfolgreiche Zusammenarbeit. Ein erfolgreicher Verkäufer sieht einen guten Kunden jahrzehntelang jede einzelne Woche, um mit ihm die aktuellen Themen oder Herausforderungen zu besprechen und zu lösen. Ebenso werden unsere Verkäufer intensiv auf den sogenannten Mitreisen im Vertrieb durch unsere Bezirksleiter geschult.

Jeder wird einverstanden sein, dass vom Kunden auszugehen ist. Wie machen Sie das besser als Ihre Wettbewerber? (lacht) Das werde ich hier natürlich nicht genau darlegen, was wir anders oder besser als unsere Wettbewerber machen. Aber 
ich kann Ihnen sagen, dass das Thema Kultur bei uns eine elementare Rolle spielt. Es dreht sich alles nur um die Kunden und hier geht es eben um Vertrauen, Offenheit und Ehrlichkeit. Wir wollen nicht nur einen Auftrag schreiben und dann wieder abzischen, sondern uns gemeinsam mit unseren Kunden über Jahre oder sogar Jahrzehnte entwickeln.

\section{Welche Hürden bzw. Herausforderungen treten dabei auf?} Wir mussten und müssen natürlich auch umdenken. Da gibt es schon andere Konkurrenten, die alles nur billig und unpersönlich über das Internet verkaufen. Aber das ist dann eben nicht Würth und das wollen wir auch nicht. Trotzdem spielen die einzelnen Kunden-Kontaktpunkte eine wichtige Rolle. Es gibt Produkte, die keine Beratung benötigen und ganz leicht online bestellt werden können, andere will der Kunde vor dem Kauf einmal in der Hand halten. Dafür eignen sich unsere Niederlassungen perfekt. Für komplizierte Themen oder auch neue Produkte sind ganz klar unsere Verkäufer gefordert. Diese Klaviatur muss ein erfolgreicher Verkäufer eben jeden Tag spielen und beherrschen.

Wie denken Ihre Verkäufer - und interne Personen - neu? Was sind die wesentlichen Vorteile von Value Selling? Das persönliche Verkaufen hat sich, bis auf die Werkzeuge dazu, nicht geändert. Es wurde nur über die Jahre komplexer, professioneller und vielseitiger.

\section{Welche positiven Effekte und Erfolge hat es gegeben?}

Das ist ganz einfach zu beantworten: mehr Umsatz, Wachstum und Expansion.

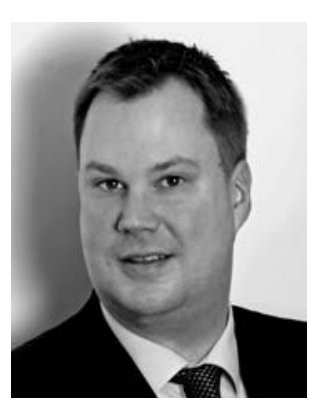

\section{Christian Peters}

Mercuri International Deutschland GmbH, Meerbusch,

\section{Deutschland}

E-Mail: christian.peters@mercuri.de
Verwenden Sie Kundenfälle auch im Marketing und Verkauf?

Ja, natürlich. Aber auch hier geht es wieder um das Thema Value Selling. Die Kunden freuen sich, wenn Sie durch uns mehr Reichweite und Beachtung erhalten und wir sind froh, wenn wir bei absoluten Profis mit unseren Produkten und Services gut ankommen.

\section{Lernen Sie auch aus den Misserfolgen?}

Natürlich. Das ist unabdingbar wichtig. Wir optimieren uns stetig.

Inwieweit "spielen“ die anderen Abteilungen zusammen mit dem Vertrieb das Value-Selling-Lied?

Alle Prozesse entlang der Wertschöpfungskette im Unternehmen müssen auf die Kundenbedürfnisse ausgerichtet sein und dem Kunden einen Mehrwert bringen.

Was scheint Ihnen besonders kritisch, um zukünftig noch mehr Erfolge mit Value Selling zu erzielen?

Es ist ja schon so, dass wir auch die Konkurrenz spüren und alle mit billigsten Preisen das Internet fluten. Aber genau hier liegt die Kunst des Direktvertriebs: zu erkennen, welchen Kunden man über welchen Kontaktpunkt mit den richtigen Maßnahmen anspricht, damit dieser Service auch als Mehrwert beim Kunden gesehen und angenommen wird. Hier muss durch den Verkäufer und das Unternehmen Vertrauen aufgebaut werden.

\section{Unternehmen}

Die Adolf Würth GmbH \& Co. KG ist das Mutterunternehmen der global tätigen Würth-Gruppe. In ihrem Kerngeschäft, dem Handel mit Montage- und Befestigungsmaterial, ist der Konzern Weltmarktführer. Die Würth-Gruppe besteht aktuell aus über 400 Gesellschaften in mehr als 80 Ländern und beschäftigt über 69.000 Mitarbeiter. Davon sind 31.000 fest angestellte Verkäufer im Außendienst. Im Geschäftsjahr 2015 erzielte die Würth-Gruppe gemäß vorläufigem Jahresabschluss einen Umsatz von 11,05 Milliarden Euro. www. wuerth.com 




